\author{
Preprint: Please cite as: \\ Blandford, A., Faisal, S. \& Attfield, S. (2014) Conceptual Design for Sense Making. In W. \\ Huang (Ed.) Handbook of Human Centric Visualization. Springer. 253-284. DOI
}

10.1007/978-1-4614-7485-2

\title{
Conceptual Design for Sensemaking
}

\author{
Ann Blandford ${ }^{1}$, Sarah Faisal ${ }^{2}$ and Simon Attfield ${ }^{3}$
}

\begin{abstract}
The focus of sensemaking research is often on process and resources such as "schemas" and "frames". Less attention has been paid to the conceptual structures that make up the schema or frame, or how visualisations can be designed to support users' conceptual structures. In this chapter, we present an approach to gathering user requirements based on the conceptual structures that people are working with when making sense of a domain. We illustrate the approach with examples drawn from our own experience of designing, prototyping and testing an interactive visualisation tool for making sense of academic literature and of studies of sensemaking by lawyers and journalists. We discuss how to move from requirements to design, drawing on a classification of visualisations that highlights their principal conceptual structuring basis. Since each individual makes sense in their own way, it is beneficial to include features that enable people to work with a representation in their own way; for this, appropriation tools are helpful. We discuss the design of such features. Finally, we present an approach to evaluating interactive visualisations in terms of their support for sensemaking, focusing on the quality of the fit between users and system.
\end{abstract}

\section{Introduction}

One of the important roles for visualisations is to support sensemaking, as people can often assimilate information much more rapidly through visualisations than through text [39]. In designing visualisations for sensemaking, however, relatively little attention has been paid to users' conceptual structures, or how visualisations can be designed to support those structures. Similarly, sensemaking research and practice has largely been on process and resources: on the pattern of activities involved in sensemaking and on the kinds of

\footnotetext{
${ }^{1}$ Ann Blandford

UCL Interaction Centre, University College London, Gower Street, London, WC1E 6BT, U.K., e-mail: a.blandford@ucl.ac.uk

${ }^{2}$ Sarah Faisal

UCL Interaction Centre, University College London, Gower Street, London, WC1E 6BT, U.K., e-mail: sarah.fba@gmail.com

${ }^{3}$ Simon Attfield

Middlesex University, School of Engineering and Information Sciences, The Burroughs, Hendon, London, NW4 4BT, U.K., e-mail: S.Attfield@mdx.ac.uk
} 
intermediate representations that people typically work with. The focus of this chapter is on how an understanding of users' conceptual structures can inform the design and evaluation of visualisations to support sensemaking.

We present an approach to gathering user requirements based on constructing an understanding of the conceptual structures that people are working with when making sense of a domain. In the following sections, we discuss how to gather user requirements for sensemaking visualisations, how to design suitable visualisations, and then how to evaluate those visualisations with users. This is grounded in our own experience of designing, prototyping and testing $\mathrm{ALVi}$, an interactive visualisation tool for making sense of academic literature $[17,18]$ and of studies of sensemaking by lawyers and journalists [3].

\section{Background}

Information visualisations are tools that interactively generate and display visual representations of abstract domains. As users interact with the tool, "ah HA!" moments arise [33]. Recognising the power of externalisations, Ware [39] proposes that visualisation has moved out of the mind and onto the computer screen. This may be true from the designers' perspective, but for users, sensemaking is very much in the mind. As users interact with the externalisations, internal conceptualisations of the domain are created, updated and used [30]. The challenge for design is to create interactive visualisations that really support their users in making sense of the domain in question.

When it comes to the design of information visualisation tools, guidance exists for determining the visual attribute (e.g., color, shape, or size) that best communicates specific types of information (e.g., variance, type and extent) [5,37]. There are also guidelines such as Shneiderman's [31] visual information-seeking mantra and its associated design guidelines: overview, zoom and filter, details on demand, view relationships, history and extract. These address the functionalities supporting the exploration of the visualisation. In addition, Craft and Cairns [12] list resources that information visualisation designers often rely on, namely design examples, taxonomies, guidelines and reference models. When it comes to designing the visualisation as a whole, though, there is little clear guidance on how to create the best possible conceptual structure; this is the focus of this chapter.

The core conceptual structure of the design is the same for all users, but there is also scope for creating individualisation features to accommodate individual differences. A further angle to our story is that not every user of a visualisation understands it or uses it in exactly the same way, so it is also important to support these individual differences as far as possible. The approach that we propose for doing this is by creating tools for appropriation, allowing the user to 'take ownership' of the visualisation to make it work for their own purposes.

To set the scene for this chapter, we briefly summarise relevant background work on sensemaking, on conceptual structures, and on appropriation.

\subsection{Sensemaking}

Sensemaking is generally regarded as a process through which an individual makes sense, or constructs understanding, of a domain or topic. The literature on sensemaking locates it within active information seeking and interpretation. Pirolli and Card [29] describe it as 
involving two interconnected loops of activity: information foraging and sensemaking. The foraging loop involves seeking, filtering and extracting information, while the sensemaking loop involves constructing a conceptualisation that best fits the evidence at hand. This process is individual and subjective. Using different language to describe very similar ideas, Klein et al [24] explain sensemaking in terms of a data-frame theory. In their view, when engaged in sensemaking, people explain elements by fitting them into a frame which links them to other elements that have resulted from their past experiences. These representations, whether referred to as frames [24] or schemas [30], are subjective lenses through which people view, filter and structure the data.

The classical views of sensemaking have focused on the finding, organisation and synthesis of information, and somewhat overlooked the use of the resulting understanding for example, in preparing a report or making a diagnosis. Blandford and Attfield [7] extend this to account (at least at a high level) for the use that is made of the information, through an Information Journey (see Figure 1). An information journey typically starts with either identifying a need (a gap in knowledge) or encountering some information that addresses a latent need or interest. When working with a visualisation tool, the tool use might be instigated by a recognised need, or the user might come across some information (or construct some understanding) that makes them want to know more. When an explicit need has been identified, a way to address that need has to be determined and acted on. This might involve asking the person at the next desk, looking "in the world" or interacting with a visualisation tool; very often, for a complex need, finding information will involve several different resources and activities. Information that is found often needs to be validated and interpreted (made sense of). And it will often be used in ways that then highlight further information needs.

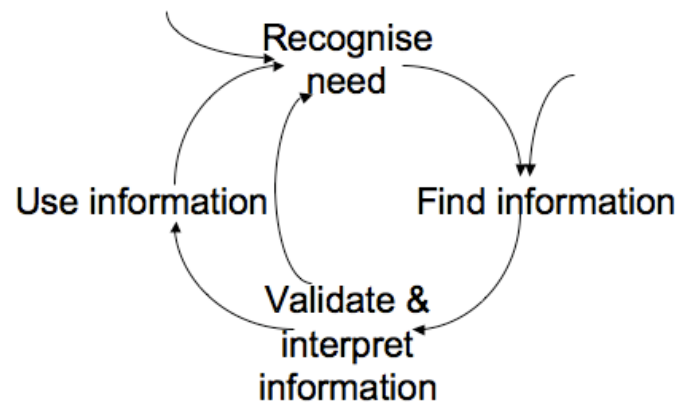

Figure 1: The Information Journey

Most current information visualisation tools address the foraging loop more than the sensemaking loop - that is: they make information available, and allow people to search it or navigate through it, but they provide little explicit support for the sensemaking activity (i.e. interpretation of information relative to current understanding). There are a few notable exceptions to this, including Jigsaw [34,35], which supports investigative analysis in fields such as law enforcement and intelligence, $\operatorname{ALVi}[17,18]$, an academic literature visualisation tool, and Aruvi [32], a scatterplot visualisation that supports analytical reasoning.

Jigsaw $[34,35]$ supports investigative analysts making sense of collections of documents that comprise evidence in an investigation. The design is based on the sensemaking literature (e.g. [29]) and has a focus on explicitly addressing user requirements. Jigsaw uses information extraction to identify entities in raw texts and then show connections between 
entities across documents. Users can view chronologies, relationship diagrams and groupings of information. An evaluative study of investigative analysis [23], which included Jigsaw as the most sophisticated of four analysis tools, provides some evidence that Jigsaw is effective, but the focus of the study was on the investigative process (for which the tools provided support) rather than on explicitly evaluating Jigsaw.

ALVi is an interactive visualisation of academic literature (see Figure 2 for an example screen) that is designed to support researchers in making sense of their literature domain [16]. The user can explore the literature using four linked views that focus on authors, author citations, publications, and publication citations. Users are also able to highlight items using a marking tool. The prototype has been implemented using the infoViz '04 dataset [19], and evaluated with researchers with an interest in information visualisation [18].

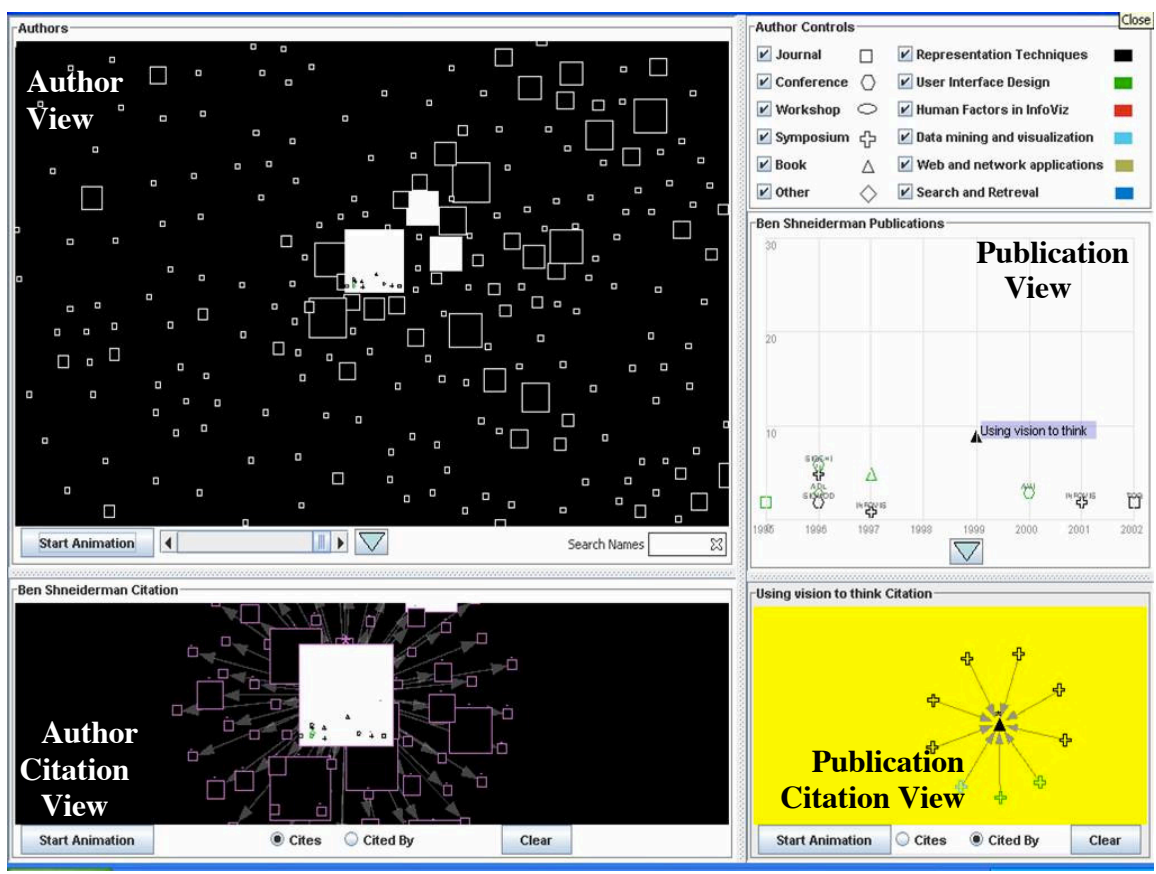

Figure 2: Screenshot of ALVi

Unlike the other two visualisations described, Aruvi [32] is intended as a generic tool to support analytical reasoning (without targeting a particular user population). The design is based on both the sensemaking literature and also the cognitive reasoning literature. The prototype implementation provides three different views: a data view (including interactive visualisation tools); a knowledge view, which allows the user to make and manage notes on the analysis; and a navigation view, which maintains a history trace of the visualisations explored in the data view. A formative evaluation, in which four participants used Aruvi on their own datasets, highlighted the value of the visualisation.

All three examples illustrate a design approach based on understanding users' needs and the ways that they think about their tasks and activities, so that the systems that were implemented support, as well as possible, users' sensemaking activities. They all implement conceptual structures appropriate to the domain, as discussed in the next section. 


\subsection{Conceptual structures}

Every domain has a structure [4] which influences the conceptualisations that users generate. Although there has been limited study of this topic over the years (e.g. [26, 28]), there has been surprisingly little work on how the conceptual structure of a design supports or hinders people working with a system. Norman [27] argued that it was the designer's responsibility to make the conceptual structure of the design clear to the user through the interface, but the converse argument - that the designer should aim to understand and actively support pre-existing conceptual structures - has rarely been made explicitly.

Johnson \& Henderson [22] echo Norman's [27] view, explaining that "A conceptual model describes how designers want users to think about the application" (p.18). It is implicit in their writing that they recognise the importance of implementing systems that support users' conceptual structures consistently and effectively. However their focus is on how to move from the conceptual model to the implementation, rather than on how users' pre-existing conceptual models might inform the designer's conceptual model.

The question of how to identify the user's conceptual model and how to assess the quality of fit between the user's conceptual model and that implemented within the system is addressed by Blandford et al [9]. They present an evaluation method, CASSM, that supports reasoning about conceptual fit between a system and its users. This approach is described in more detail below.

\subsection{Appropriation}

As well as depending on the suitability of generic conceptual structures, sensemaking also relies on the strategies that people adopt to generate personal conceptual structures. Since each individual makes sense in their own way, it is beneficial to include features that enable people to work with a representation in their own way; for this, appropriation tools are helpful. By appropriation we mean making technology 'one's own' in ways that the designer could not anticipate; for example, features for labelling or placing objects (physical or digital) are used in widely differing ways by different individuals and groups.

Designing for appropriation [13] allows people to express ownership of the represented problem domain in ways not anticipated by the designers. Appropriation concerns the ways in which technologies are adopted, adapted and shaped by their users [10]. Dix [13] proposes several guidelines for appropriation:

- Allow interpretation (e.g. providing colours or annotation features that enable people to assign their own meanings to the marking).

- Provide visibility: making the system state clear so that people can infer when they need to find a work-around (e.g. because an important feature is not functioning properly).

- Expose intentions: making the designers' intentions clear to the users so that they better understand when workarounds are and are not appropriate. The example Dix gives is of login procedures, which might be to provide security (which could be provided some other way) or to provide personalisation features (so that login is about identification, not just authorisation).

- Support not control: making it possible for people to vary routines in common tasks while nevertheless keeping them efficient.

- Plugability and configuration: creating systems that can easily be reconfigured by users.

- Encourage sharing: providing ways for end users to share tips on how to use a system. 
- Learn from appropriation: develop new generations of technology that better support the new use (Carroll and Rosson [11] discuss this as a "task artefact cycle").

In the context of visualisations to support sensemaking, probably the most relevant and valuable of these guidelines are providing support for interpretation (as implemented, for example, in Aruvi's annotation feature and ALVi's marking tool) and ensuring that the system provides support, not control, so that people can explore data in ways that suit them.

\section{Gathering requirements}

In order to understand how people think about a domain (the world of which they are trying to make sense), it is necessary to work with those people, listening to their descriptions of their world. For example, in our studies of academics' interactions with their literature [16], we found that ideas and authors (i.e. other researchers) are more important than particular papers; in a study of lawyers [3], we found that people and events were central to the building of chronologies (or causal narratives), which had to be backed up by evidence (typically in the form of documents and records such as telephone call logs).

There is often a tension between the way a user naturally thinks about their problem and concepts that can be implemented in a computer system. For example, in ALVi it was straightforward to represent the concept of authors of papers, but much more difficult to represent the concept of ideas, which are meaningful to users but are not directly represented within the text of a paper. Conversely, in implementing a system it may be possible to make new concepts available to the user that are actually valuable to them, and that extend their space of thinking. For example, when the first author started blogging, she had not considered what factors might make one post more or less popular than another, but the visualisations of statistics made available through the blogging tool encouraged consideration of how to publicise the blog posts, when people typically access the posts, and why some posts might be much more popular than others which, over time, informed her approach to writing and publishing posts.

There are many ways of gathering user requirements, but at the centre is a need to engage with the potential users of a system, to get a deep understanding of their goals and activities, and the ways that they think about their sensemaking challenges. There are entire textbooks on gathering user requirements (e.g. $[6,20,25])$, and it is not possible to do justice to all possible approaches within one chapter, so here we focus on techniques that are particularly pertinent for designing sensemaking visualisations. In particular, to gather information about people's conceptual structures, it is necessary to gather verbal data. So we focus on interviews and Contextual Inquiry as ways of gathering data, and then briefly outline one qualitative approach to analysing that data.

\subsection{Interviews}

Interviews may be more or less structured. For gathering requirements, semi-structured interviews are generally the most effective. Such an interview allows the interviewer to ensure that they cover the important questions (e.g. about what people are trying to achieve, what they do, what is important to them) while also having the opportunity to pursue unexpected interesting avenues as they arise. 
Key steps to planning interviews are:

- Planning questions

- Identifying and recruiting participants

- Deciding on practicalities such as locations, times and lengths of interviews

- Deciding how to record and analyse data

When planning questions for designing visualisations for sensemaking, the heart of the interview is likely to be around how people make sense of the domain of interest, and what questions they have about it. For example, when designing ALVi, we wanted to know how young researchers went about identifying and familiarising themselves with the research literature in their field, and how more senior researchers thought about the literature in their field, and how they might go about learning about a new literature (e.g. if shifting fields).

Every interview has a beginning, a middle and an end. The aim at the beginning is to set the scene, to put the participant at ease (e.g. that there are no wrong answers, that they are the experts in the domain, that their participation is valuable and will inform the design of the proposed system), and to elicit basic background information (e.g. what their job function is, how long they have been active in this area). The aim of the middle is to build a rich understanding of their work and their sensemaking activities, while the aim of the end is to tie up any loose ends, thank the interviewee and close the interview.

Interview questions should, as far as possible, be short and to-the-point, using familiar language. They should not be leading (i.e. suggesting a particular answer), but should be as open and unbiased as possible. During the interview, it is often helpful to reflect back to the participant your understanding of what they have told you. Probably the most important questions, though, are ones that elicit detail: not general "what do you do?" or "what are the key factors that you take into account when...?", but "can you talk me through the last time you did...?" or "was there a particularly memorable occasion when...? Tell me about it." The details of a particular incident will bring the subject to life in a way that no amount of generalisation ever can.

When determining who to interview and how to approach them, there are both ideal and pragmatic considerations. Participants should be representative of the population for whom you are designing; maybe they are existing users of a related technology or potential users of the proposed visualisation tool. There may be typical users or users who are extraordinary in some way (e.g. recognised experts or people who struggle to do the job). There may be sub-populations within the overall population that need to be catered for. There may be people who are articulate or opinionated or quiet. In an ideal world, participants will be broadly representative of the intended user population. In the real world, where it might be difficult to recruit the ideal set of participants, it is sometimes necessary to work with more of a "convenience sample" of people who are willing and able to take part; in this case, it is important to be alert to possible biases in the data, and to find ways to limit them, and to validate the findings.

There are many possible ways of recruiting participants: within an organisation, this might be largely by word-of-mouth, or internal advertisements; externally, it might involve working through special interest groups, using a specialist recruitment agency or using social media to solicit participation.

People may participate for many different reasons: maybe you are offering a material reward (participation fee, a small gift, or entry into a prize draw); maybe they are inherently interested in the topic and value the chance to discuss it and reflect on it; maybe it is important to them that they have a chance to influence a new design; or maybe they have been asked to participate by their line manager (and are therefore doing it as part of their work). It is important to recognise and work with these alternative motivations in recruiting participants and in valuing their contributions. 
You need to plan where and when to interview people. Is it important that you are physically co-located, or can the interview be conducted equally well by phone or over the internet? There are potential advantages to being co-located, including ease of establishing rapport and of sharing externalisations (e.g. sketching together or being shown relevant artefacts). Conversely, meeting up often requires that one person travel and that a suitable meeting space be available. The space should be comfortable, quiet and private enough to conduct the interview without significant distraction. Interviews in the place where people normally engage in the sensemaking activity may allow participants to illustrate activities or use resources from the environment to animate the discussion.

How to record data? This may depend on factors such as noise, confidentiality and privacy, and the forms of analysis that are planned. Sometimes, just pen and paper (or typed notes) are sufficient, or all that is possible in the circumstances; this limits the amount of subsequent transcription or analysis needed or possible. Going to the other extreme, a full video recording may capture details that would otherwise be missed, but setting up and using a video camera can be intrusive and distracting, and the benefits rarely outweigh the costs unless there is important action to record. Most often, an audio recording (which can be fully or selectively transcribed), and optionally still photos or sketches of key artefacts, achieves the best balance between richness of data and cumbersomeness of gathering and analysis.

Interviews are best conducted as conversations: asking questions naturally, and in a logical sequence (which may not be the order planned), showing an interest in the participant's accounts and stories, and valuing their expertise and insights.

One great advantage of interviews over observations is that they elicit people's understanding of the task or activity, which may be partly decoupled from the way they use a particular system to perform it. For example, someone talking about planning a holiday will probably talk about many factors that they use to decide where and when to go (the likely weather, the activities or attractions, the ease of getting there, etc.); and observation might focus on how they use flight booking websites, which will naturally highlight certain factors (e.g. cost of flights) while omitting others.

Conversely, interviews elicit perceptions, and can be unreliable for eliciting certain kinds of facts. For example, people's accounts of the steps they take to perform a task may omit steps that are so "obvious" that they are overlooked, and some information may be so taken-for-granted that it is never mentioned.

There are two widely used approaches to gathering observational data that also includes people talking about their activity. One in think-aloud [14], which can support incremental redesign (fixing limitations of an existing system), but is less suitable for identify user requirements for a novel system; we discuss think-aloud in section 5 . The second is Contextual Inquiry, which focuses more on the broader activity than on the details of an interaction, and hence is better suited to gathering requirements.

\subsection{Contextual Inquiry}

Contextual Inquiry (CI) is described in detail by Beyer and Holtzbatt [6], so here we provide just a brief overview of the approach. It is a form of data gathering and analysis that takes place in the work setting (or wherever the activity naturally takes place). It involves observation of the activities of interest, and questioning about those activities.

Just as, when discussing interviews, we emphasised the importance of eliciting concrete examples, not just generalisations), so in CI there is a focus on concrete data: on real artefacts that support the activity, and events that occur. Similarly, just as interviews should 
value the expertise of the interviewee, so CI values the expertise of participants in doing their work, and data gathering involves working with people on understanding their work experience. In most contexts, $\mathrm{CI}$ is conducted by a team of people, each gathering some data and then sharing insights and reaching a shared understanding; for the purposes of this chapter, we focus on what each individual analyst does within that process.

At the heart of $\mathrm{CI}$ is observation followed by questioning, to develop a rich understanding of what people are doing, why, and how they think about their activities. For sensemaking visualisations, key questions will include what questions people have, what information they draw on to address those questions, and how they think about the topics they are working on.

Many of the decisions (e.g. about who to work with and how to record) are very similar to those involved when planning interviews. One important difference is that CI should always take place in the workplace (or other place where the activity is performed). There may be more compelling reasons for having a video record for $\mathrm{CI}$ than for an interview, but costs and benefits still need to be traded off against each other.

One challenge is sometimes to persuade people to continue working as naturally as possible, as some prefer to stop and chat about work rather than performing work (which makes data gathering into an unstructured interview rather than a CI). It may also be important to consider the timing of questions to minimise disruption. A further consideration in the workplace is how to fit in: are there particular dress codes? How to take breaks? How to introduce yourself to "third parties", particularly if your participant has a role where they interact with many other people? There is not a single answer to such questions, which will depend on the situation, but they should be considered carefully.

Once data has been gathered, a full CI analysis involves representing the findings in terms of five models:

- A flow model, which focuses on the flow of communications around the organisation (between people mediated by artefacts).

- A sequence model, which focuses on the order of events.

- An artefact model, describing the physical structure and noting reasons for that structure.

- A cultural model, noting roles and relationships.

- A physical model, noting influences of the environment on performance.

The process of creating representations, as well as the results, provide a focus for thinking about new design solutions. However, when it comes to designing sensemaking visualisations, much of the information represented in the recommended models is of limited value and relevance, so in the next section we present an alternative approach to analysis that focuses on conceptual structures.

\subsection{Identifying users' conceptual structures}

As discussed above, there are many kinds of user requirements, and in this chapter we are focusing on understanding the conceptual structures people work with, and how to translate that understanding into requirements for design. When evaluating a particular system, one can go further and assess the quality of the conceptual fit between user and system - a topic to which we return below.

A concept is a "thing" or a "property" that the user works with while making sense of information. These can be divided into entities and attributes, although early on in analysis, 
this distinction may not be important. An entity is often something that can be created or deleted within the system. Sometimes, entities are things that are there all the time, but that have attributes that can be changed. In ALVi, entities include authors and publications. An attribute is a property of an entity. For example, an author has a number of publications, signified in ALVi by the size of the rectangle representing the author. There may also be relationships between entities - for example, that one paper cites another paper.

Having gathered user data as described above, one way to conduct an analysis is to go through the words (e.g. transcription of users talking or documentation) highlighting nouns and adjectives, then deciding which of those words represent core concepts within the user's conceptualisation of the domain.

Depending on what matters most, the analyst might distinguish between entities and attributes to achieve clarity in the model. Concepts might also be grouped into related ones that function together, or that might be displayed together. Relationships between concepts also need to be noted.

To illustrate the approach, we take brief quotations from the requirements study that led to the development of ALVi and highlight key concepts and relationships. This analysis is described more fully by Faisal et al [16].

Participant 3 said: “...there'll be a core body of people who are aligned with particular kinds of ideas". This highlights two key ideas: a body of people (which in turn comprises individual people) and ideas. It is unclear from the way participant 3 talks whether the body of people might also be called a community, or whether it is better regarded as a group within a community. Further analysing the data, we decided that this distinction did not matter, as the concept of a community is only loosely defined anyway.

Participant 6 also highlighted the importance of ideas: “...I think I would go for ideas...what it means actually it is not the paper but the ideas". Ideas are presented in papers. In turn, papers are written by authors, as participant 2 stated: "It is hard to separate that [papers] from authors, 'cause ultimately they were written by authors". Note that different terms (people, authors, also researchers) were used by participants in the study to refer to the same concept (namely the people who do research and then write it up).

Participant 3 identified an attribute of a paper that was important, namely its influence: "I could name you papers that have been influential ... in terms of changing thinking in a particular area". As well as particular papers, there are also authors who are influential within a community, as participant 5 noted:“... you have to read what they [influential authors] are doing even if you don't agree"

The word "influential" was used in two different senses. As well as being recognised as being influential by a community, some participants recognised a more personal kind of influence. For example participant 3 said: “...there have been papers that have been influential ...actually changed the way I have thought of my work". Similarly, participant 4 noted that: "I suppose when you say influential I consider it to be influential to my own ideas". This difference between influence on the community and personal influence appeared to be important. The first might be represented indirectly by number of citations, whereas the second is internal to the user, and might be externalised (e.g. through the use of the marking feature mentioned earlier).

In summary, from these selected extracts we have identified important user entities of person (with attribute community influence), community, idea, paper (with attributes community influence and personal influence), and relationships that person is-member-of community; paper is-written-by author; paper encapsulates idea.

In this particular domain, relationships are important, and chronology (how a field develops over time) may also matter, so visualisations have to be designed to allow people to explore relationships and chronology. The design of ALVi is based on these principles. Other domains have different central properties that should be designed for, as discussed in the next section. 


\section{From requirements to design: a space of sensemaking visualisations}

In moving from requirements to design, it is necessary to determine what kinds of visualisations will best support people's sensemaking. Here, we propose and illustrate a classification of visualisations that highlights their principal conceptual structuring basis, based on work reported by Faisal et al [15]. This classification (into spatial, sequential, networks, hierarchical, argumentation and faceted) reflects the different conceptual structures of different sensemaking domains.

For each visualisation structure, we propose a scenario of use for which that structure would be appropriate, and highlight key properties of the structure.

\subsection{Spatial}

Spatial representations depict objects and their spatial relationships. In such a representation, elements such as orientation, distance and location are critical components. Visualisations based on maps, such as geographical information systems, are typical examples of spatial visualizations. They support planning that is based on spatial attributes (such as planning a trip), and reasoning about properties that are based on space or geography, such as the spread of disease or the distribution of wildlife across a region.

\section{Scenario: Planning a Trip}

Samantha is planning a trip from London (UK) to South Africa. She has decided to visit Cape Town, Pretoria and Johannesburg. She checks out a travel website and determines that both Cape Town and Johannesburg have international airports, but that Pretoria does not. Her task now is to determine a traveling route. In order to do so she consults a map as she has only a vague memory of the layout of South African cities. She sees that Pretoria and Johannesburg are towards the north east whilst Cape Town is further south. She also notes that the distance between Cape Town and Johannesburg is too great to be covered in a day except by flying, whereas Johannesburg and Pretoria are within comfortable driving distance. From her previous spatial knowledge, she knows that London is far north. It will take her longer to travel from London to Cape Town. She decides that she would prefer to make the longer journey on her way to South Africa rather than on her way back to the UK. Therefore, she settles on the following travel route: fly from London to Cape Town and from Cape Town to Johannesburg, drive to Pretoria then back to Johannesburg in order to catch a plane back to London.

A simple design example to demonstrate the spatial representation is Google Maps (Figure 3). As part of this application users are able to locate themselves in terms of the map's spatial layout, get directions to wherever they want to go and determine areas with high traffic which assists then in planning their journey. 


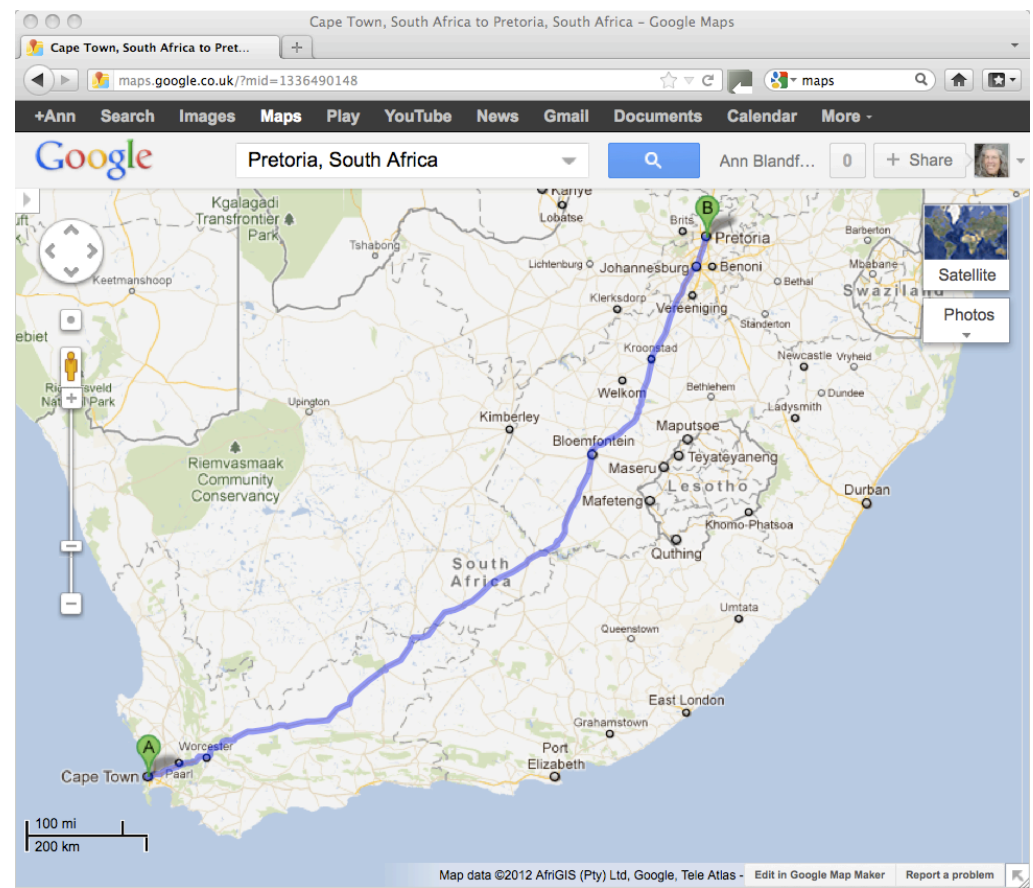

Figure 3: Spatial visualisation

\section{Properties}

Two and three dimensional spatial representations are generated in order to make sense of physical layouts. Information such as the relative and absolute locations of different kinds of objects is crucial and so these are generally mapped proportionally within the representation. Spatial representation can also be used to represent a domain metaphorically. For example, our own discussions with a programmer indicate that the way he makes sense of code is by conceptualizing it spatially. Spatial representation offers a means of depicting complex abstract relationships in a simplified way.

\subsection{Sequential}

Sequential representations depict movement through a series of elements based on a predefined order such as time. Chronologies are a common example of sequential representation, exemplified for example in legal investigation support software such as LexisNexis CaseMap (www.casesoft.com), in which elements are events connected within a time-series. Previous field research by Attfield and Blandford [3], which looked at collaborative sensemaking activities during large corporate investigations, found that chronologies, painstakingly constructed by large teams of lawyers, provided central visual representations for sensemaking. Legal investigations extend over time, are resource 
intensive, and require the sifting and re-representation of very large collections of electronic evidence. Using the chronologies that they created, teams of lawyers could review the underlying narrative of their investigated domain, identify periods of key concern or activities of protagonists that seemed odd and potentially suspicious and, using this, refine their investigation questions and searches in ways that were more focused and tractable. This could be understood as comprising phases of data focusing (identifying and structuring information to draw out facts relevant to a given set of investigation issues), followed by issue focusing (revising the issues and questions in the light of new insights).

\section{Scenario}

Carol is a legal investigator leading a team in an investigation into potential hidden liabilities within a large multinational organisation. Carol's team has been tasked with making sense of activities surrounding a particular contract that the company had and a particular individual who led the contract. As they review hundreds of recovered emails that have been returned by searches they have constructed, they select those that seem most relevant and use these to construct a timeline of how work on the contract unfolded. They look in detail at the run up to the contract bid submission and see something unexpected. During this period which, as one would expect, is particularly busy, the manager with responsibility for the bid sends an email saying that he will be flying out to a foreign capital for a short period and flying back home again. There is no explanation for why he is going. This is odd since the lawyers would expect that during the bid preparation he would remain close to the team he is leading. This appears potentially suspicions. These scenarios typically require 'all hands to the pump'. Carol's team mention what they have found at a daily investigation review meeting and ask whether anyone has anything that may shed light on it. Another team involved in the investigation say that they have evidence of activity in the same foreign capital on that same day. The two groups align their chronologies. Carol's team see that a representative of the company visited an overseas airport that day, signed a contract, and then returned directly home. They search for the contract and find that one of the signatories was the manager in question.

Legal investigations are one scenario in which temporal representations are particularly important. Other representation are used, but chronologies often provide a central representation for reasoning. Since lawyers expend considerable energy in filtering, selecting and structuring otherwise unstructured evidence collections (such as emails) into temporally ordered narratives, they would benefit from visualisations that can automatically perform that kind of visual structuring for them. This was the rationale behind a representation called ThreadsVI [2] which aimed to associate emails in a meaningful way by representing them visually in the context of their discussion threads and in terms of senders and receiver such that the user could gain informal visual impressions of levels of activity (similar to centrality) within a semantically filtered social network over time (Figure 4).

\section{Properties}

Sequential temporal representations lay events or entities in order of, or in proportion to, separation on a temporal variable. Since sequence may demand only one dimension within a $2 \mathrm{D}$ or $3 \mathrm{D}$ visual representation, further dimensions may be used to represent other parameters in multivariate data that are salient to the usage scenario. Colour and shape can support the encoding of further variables. 


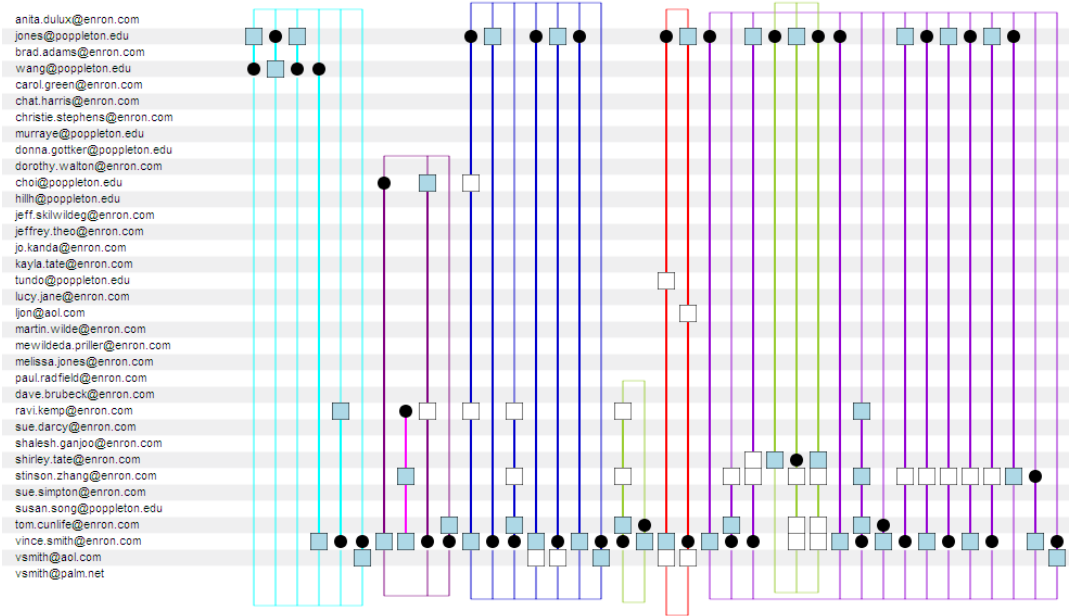

Figure 4: ThreadsVI [2], a temporally organised representation of protagonist activity within an email collection.

\subsection{Networks}

Network structures are relational structures where items may be linked to an arbitrary number of other items in many-to-many relationships [31]. These representations, for example, can support the understanding of complex social structures, e.g. Vizster [21], a visualization that allows for the exploration of online communities. ALVi's author view is another example, in which authors are clustered based on the collaboration relationship. Authors that have collaborated together will be seen as being grouped in a cluster. Based on such clustering needs, the layout of the authors view takes the form of a force-directed layout graph (Figure 5).

\section{Scenario}

Tessa is new to social media networks. She recently created an account on Facebook and added her friend Mia as a friend. Tessa and Mia have been friends since high school. They also went to college together. Hence, they have a lot of friends in common. Through Mia, Tessa started to identify friends and add them to her list of friends. Similarly through those friends she started to identify more friends and acquaintances and started adding them. Slowly Tessa was creating a network of friends whereby each person on that list was connected to her and to other people in her list of friends. As she was doing that she started to realize that she had several networks being created. For example, a network that contained mainly school friends and another that contained work friends. As she was browsing her list of fiends she realized that Anna a colleague of hers at work had a common friend, Emma. Emma was Tessa's friend in school. When Tessa asked Emma about this she realized that Anna and Emma had taken a summer course together and had been friends ever since. 


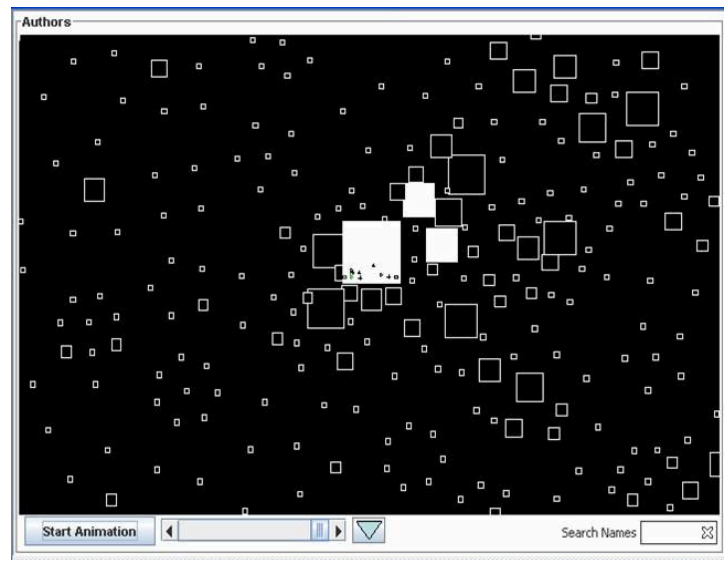

Figure 5: ALVi Authors' view

Figure 6 shows a Facebook tool that visualises a friends network. It takes all the friends, for example Tessa's friends, then links and groups them together to form a colourful image. Each node represents a friend. When a line links two friends, it means that they are friends with each other, as per the example above, Anna and Mia.

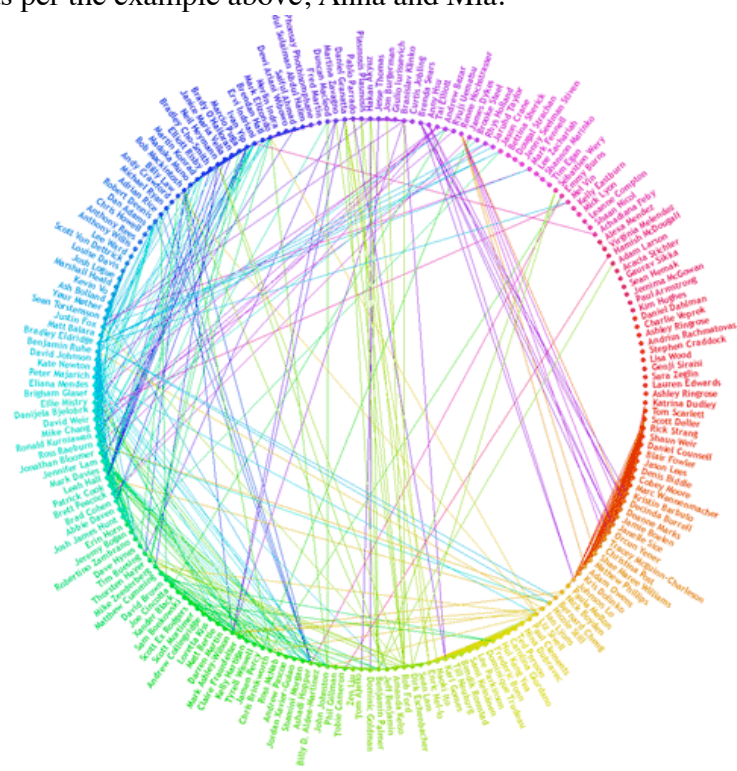

Figure 6: Friend Wheel

\section{Properties}

Network representations represent the interconnection of various items. They have a general graph structure with nodes and edges. Each edge connects two nodes. Nodes in a network can be connected by an unlimited number of edges, representing many-to-many 
relationships. Network representations are applied to a variety of domains including, but not limited to, social networks.

\subsection{Hierarchical}

Hierarchical representations model a domain by organizing elements according to asymmetric, one-to-many relations.

\section{Scenario: Categorizing Research Areas}

This scenario is based on the second author's experiences. Sarah is working in the area of academic literature visualization and would like to develop a scheme that she can use to structure her literature review. She works with a number of ideas which relate the papers in different ways, but there is no organization that seems to include all the work she wants to include in a neat way that she feels she can structure a narrative around. She considers different facets that seem to distinguish the papers. She realizes that you can describe all of the information visualization tools she has read about as falling into one of two categories: knowledge domain visualizations (KDViz) and Information Retrieval (IR) tools. This strikes her as a candidate for her high level organization. Then, as she looks more closely at the papers that fit into the IR tools' category, she sees that roughly half are concerned with interactivity and half are concerned with usability. She settles on this as her first plan for an organizational scheme (shown in Figure 7).

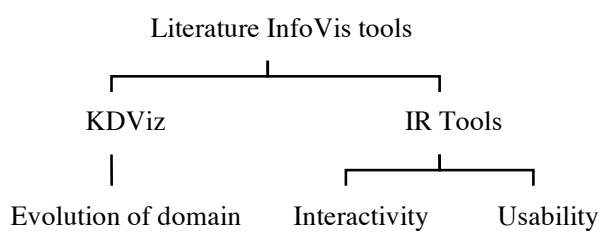

Figure 7: Hierarchical categorization

An example of an online visualization is provided by Microsoft Academic Search's representation of supervisor-student relationships, which show "genealogies" of researchers, as illustrated in Figure 8. 


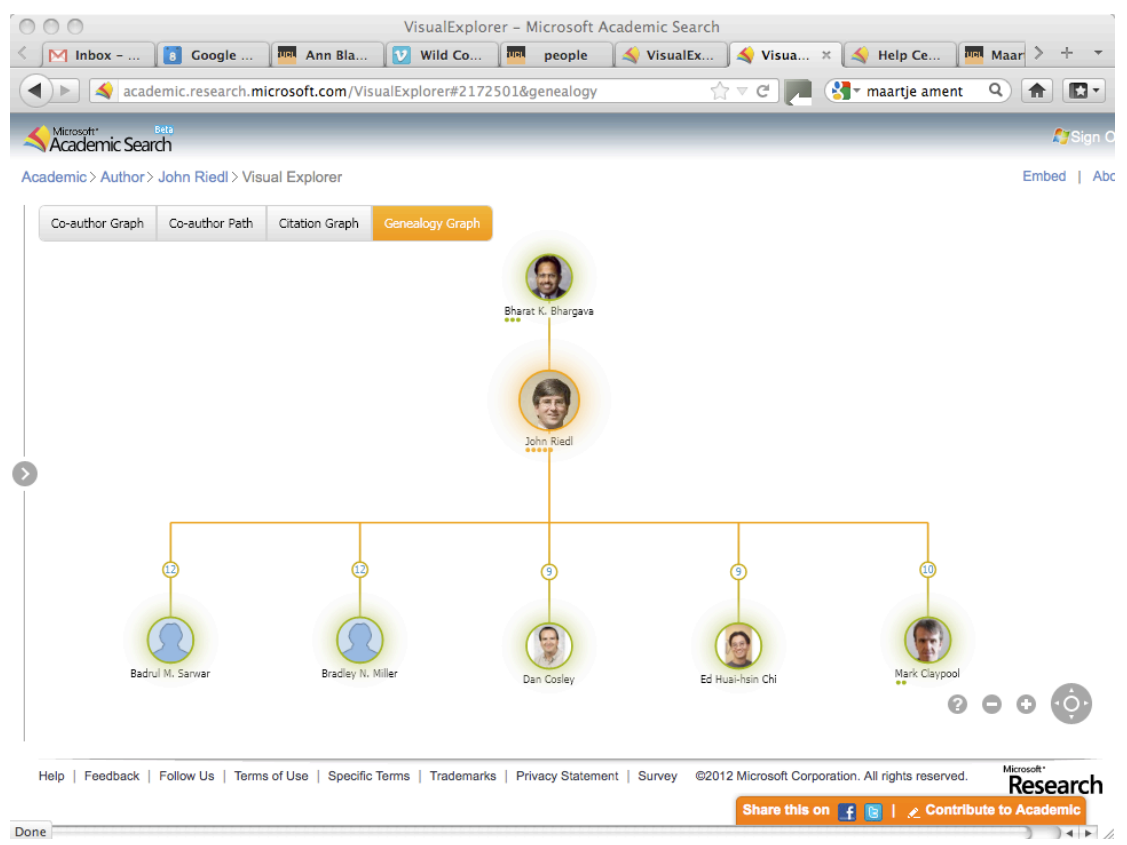

Figure 8: example hierarchy from Microsoft Academic Search

\section{Properties}

Hierarchical representations may be represented as trees [31], where each item has a link to a parent item except for the root. The relations used in hierarchical representations are often taxonomic. However, they can also be used to represent non-taxonomic relationships such as part-whole, or parent-child.

\subsection{Argumentation structures}

Argumentation representations relate multiple propositions or ideas together through argumentation operators in a way that makes inferential relationships explicit. Visual representations of arguments have a long history. For example, in the early twentieth century, Wigmore developed a visual language for representing and analyzing arguments in legal cases [40]. The Wigmore diagrammatic convention was designed specifically for representing competing arguments in a contentious, legal setting. The unit of analysis in a Wigmore diagram is the evidential proposition or statement, with these laid out in relations of inferential support. Notably, Wigmore included conventions for representing aspects of two competing arguments which you would expect to be salient to a legal mind trying to make sense of them, evaluate their relative merits, and potentially develop a legal strategy. For example, evidence is visually coded as to whether it is testimonial (stated by a witness), circumstantial (requires inference), explanatory (reduces impact of testimonial or circumstantial evidence) or corroborative (supports testimonial or circumstantial evidence). 
Evidence is also represented in terms of the side who offered it (prosecution or defense). A strength of Wigmore's scheme is the representation of the perceived strength of elements of an argument and of the argument as a whole.

Whereas Wigmore was concerned with a specific domain of activity, namely legal cases, Toulmin later developed a diagrammatic convention for the representation of everyday arguments [36]. A philosopher, Toulmin was concerned that everyday persuasive arguments rarely correspond to classical models of inference, such as the syllogism. What he felt was needed was some way of representing the form of every day arguments so, for example, these could be reviewed in terms of validity. In Toulmin's scheme there are three parts to an argument: a claim (the conclusion of an argument); data (evidential support for a claim); and a warrant (a generalised assumption on which a link between claim and evidence depends). In everyday arguments warrants are often implicit. An advantage of the Toulmin approach was that by making them explicit they become amenable to consideration.

\section{Scenario: Conducting a literature review}

Paul is an HCI researcher. He is reading around the topic of information seeking looking for ideas for tools and functionalities that might usefully augment digital library systems. $\mathrm{He}$ is looking at studies of information behavior in order to gain insights about the things that people naturally do with paper documents in order to trigger ideas about how people might wish to interact with digital documents. Paul notes a study (a) which reports that people use physical piles of paper as a way of informally organizing task related information. This reminds him of a study (b) which reported on the way that paper documents on the desk are sometimes used as reminders for action. He notes that digital libraries don't provide tools that support these kinds of behaviors. Later he reads a paper (c) which describes a spatial hypertext system and how such systems allow users to create informal, visual document arrangements which can persist across sessions. Paul uses these claims to construct an argument which acts as motivation for his new idea of augmenting digital libraries with spatial hypertext functionality.

ClaiMapper [38] is an example of an argumentational representation. It allows users to create concept maps of literature by manually dragging and dropping concepts and building relationships between them. This is done with the aim of creating coherent arguments by sketching out rough structures as informally as required.

\section{Properties}

Argumentation representations are formed around the integration of a series of claims from which a conclusion is inferred. As a result, the associated tools need to allow users to build dependency connections between different parts of the data, whether these are explicitly expressed by the data or inferred. 


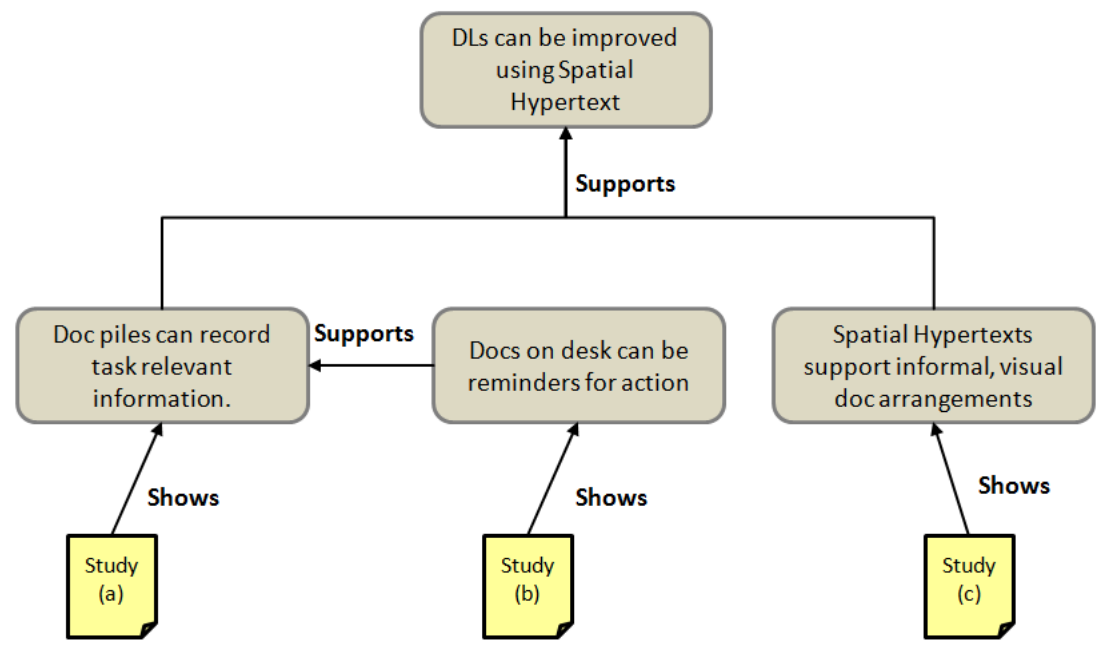

Figure 9: The Spatial Hypertext example shown in a form of argument map. Rounded rectangles represent propositions and arcs either represent relations of inferential support or links with source study reports.

\subsection{Faceted}

Faceted representations show a set of entities within a domain in terms of a set of properties. These will often also exploit other visual representations such as spatial (e.g. [41]) or temporal (e.g. [1])

\section{Scenario: Planning a trip to the cinema}

Claudia and Jeff want to watch a film. They are not in the mood for anything too serious. A romantic comedy would be ideal. However, they do not have a particular movie in mind. They start browsing online to identify a title that interests them. There are too many to choose from. Hence, they decide to select something that is quite recent. They look for films that have been released in the past two years. Lots of titles come up which sound interesting. However, when looking at the reviews, some have low and average ratings. Consequently, they decide to look for a film that has good reviews. This narrows down their choices. They settle on "Midnight in Paris" as it was a romantic comedy, released in 2011, with excellent reviews. This scenario involves relating film selection to an evolving set of facets derived by the user's changing knowledge of the various possible film criteria. 


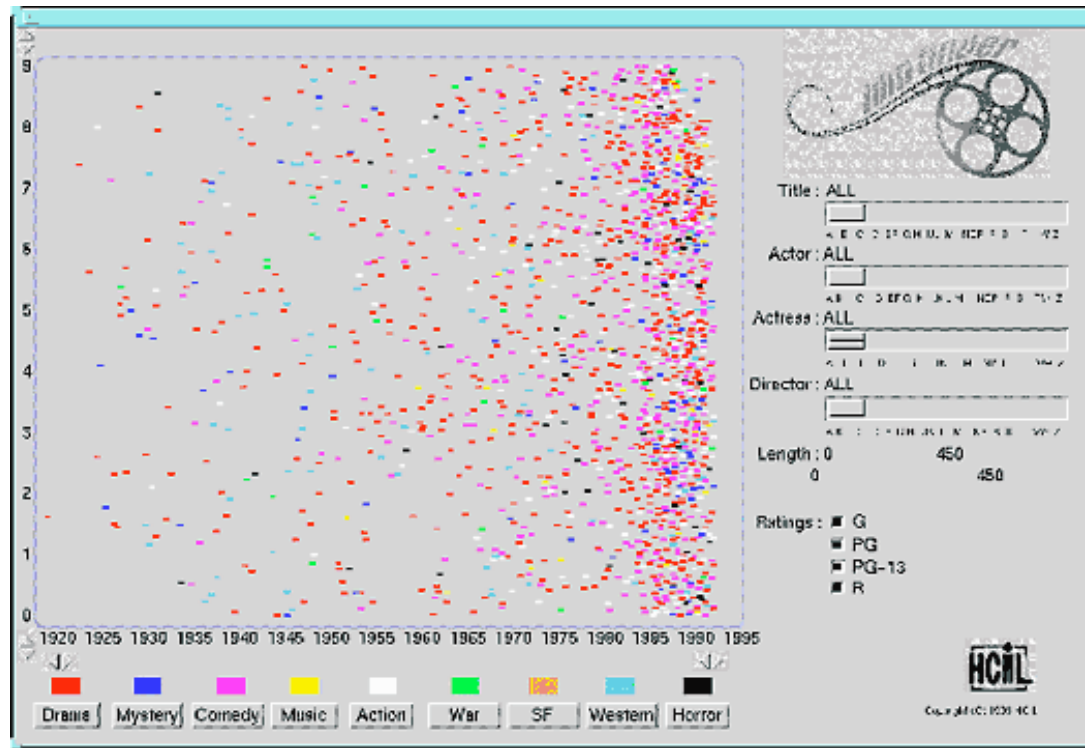

Figure 10: FilmFinder

FilmFinder [1] (Figure 10) is a visualization tool that represents films in terms of (and filtered by) their properties. In this application films are represented as colored squares, where color encodes genre (e.g. horror, comedy, science fiction, etc). The films are laid out in a scatter plot, in which horizontal position indicates year of production and vertical position indicates popularity. Sliders allow users to filter the database by different properties, e.g. title, actor, etc. The FilmFinder application can be considered as an example that supports the facets representation allowing users to compare objects according to properties that best represent their interests.

\section{Properties}

As part of the faceted representation, entities within a domain are shown in terms of a set of facets. The best possible match is used to either make a choice or provide an interpretation of a specific situation.

\subsection{Designing for subjectivity}

The scenarios presented here all reflect sensemaking activities, but they are all characteristically different. By classifying these situations based on representational structures we can effectively determine the best visual layout associated with the supporting tool. For example, in the case of Claudia and Jeff the supporting tool must capture and represent the various facets that support their film-going decision, whereas in the case of Sarah the supporting tool needs to assist her in organizing and representing the data hierarchically. 
It is important to note that the design examples presented in this paper do not take into account the subjectivity of the experience, except for ClaiMapper [38], which allows users to add links between the various concepts. We believe that designing for subjectivity is crucial when it comes to sensemaking. As discussed above, ALVi also takes into account subjectivity of the sensemaking experience. It allows users to apply their personal sensemaking needs and style through the use of the marking tool which allows users to code any displayed entity in green. No meaning was associated with that colour or action. Users used the 'marking tool' differently depending on their goals and ways of working, i.e. they appropriated it to meet their own needs. The following are a few examples of how personalisation was achieved:

- Subjectively filtering the data - U1 said: "having a sort of representation of what responded to a query term and then being able to go through them and put my own, so it's like a two step filter so the system filters and then I filter that was really useful." The user used the word "my own" to refer to his experience in using the marking tool. He indicated that he was able to further filter the data by overlaying his filtering scheme over the system's filtering by marking some of the results of the system's filtered outputs.

- Setting landmarks - U6 said in relation to the marking tool's benefits: "I won't feel lost I can go back". Similarly, U11 said: "I started marking because I looked at stuff and then I moved on and then I came back to it and I realized I actually read that but because I hadn't marked it I did not realize I had read it".

- Keeping track of the amount of work - U10 said: "I have a sufficient amount of papers actually from it being highlighted so I know that I possibly have a sufficient background for this particular reason".

- Generating personal overviews - U8 expressed it: "I remember um searching names, marking the authors and then towards the end I remembered that you could mark all the associated authors which is a really handy little thing if you want to get a grips with much of the overview much more quickly".

From these examples it can be seen that the users were able to associate different meanings that were personal to them and that fitted their own sensemaking strategies with the marking colour. This feature was successful in allowing users to implement their personal sensemaking strategies.

\section{Evaluating the appropriateness of conceptual structures}

So far, we have discussed gathering user requirements and selecting appropriate conceptual representations for the design of interactive visualisations. The third key step is evaluation of the resulting interactive visualisation in terms of its support for sensemaking. There are many possible aspects to evaluation, as discussed by Kang et al [23] and Faisal, Cairns \& Blandford [18]. For the purpose of this chapter, we continue our focus on the quality of the fit between users and system [9].

In an earlier section, we discussed how to gather user requirements and identify key concepts. Evaluation builds on those techniques, comparing the users' conceptual structures with those now implemented within the visualisation tool. This approach can be used to evaluate an existing implementation (that was not developed by starting with user concepts); in that case, it is necessary to gather user concepts as described above, even if they are being used principally for evaluation rather than for design. 
As well as the interview and Contextual Inquiry techniques described above, it may also be appropriate to make use of Think Aloud protocol at the evaluation stage, as this can highlight additional concepts that emerge in the interaction between user and visualisation system.

At this stage, we are considering conceptual fit between a user and the system: the user might have some concepts that are not represented within the system ([a] in Figure 11); conversely, there may be system concepts that the user is not familiar with ([c] in Figure 11). The concepts that are shared between system and user ([b] in Figure 11) are a conceptually good fit.

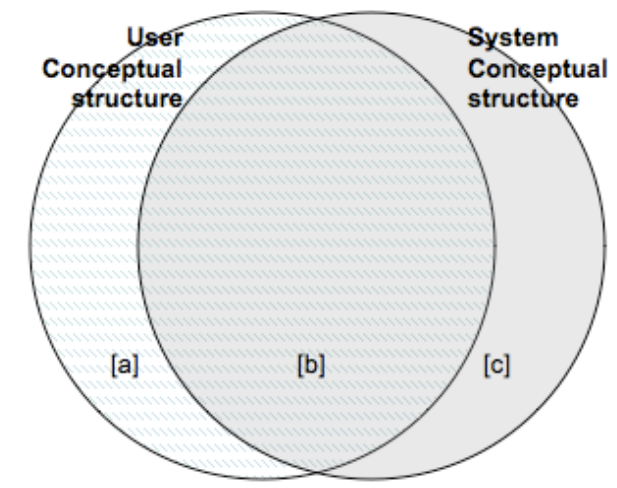

Figure 11: User-system fit.

\subsection{Think Aloud Protocol}

Think-aloud involves the user of a system articulating their thoughts out loud as they work with a system; it creates data on how people think about their activity and about the system they are working with. Think-aloud [14] involves recording and analysing people's thoughts about the activity they are currently engaged in, and typically focuses on the interaction with a current interface (and hence is well suited to identifying strengths and limitations of that interface).

If think-aloud data is being gathered to support analysis, based on how people use a current system, then it is important that the tasks given to study participants are domainrelevant, and give the participants scope for discussing domain concepts. For example, the analyst evaluating ALVi would get little useful data if the user task were given as "use ALVi to find out who has cited the work of Matthew Ward". The task description we used for evaluating ALVi was based on our original requirements study, and intended to represent a typical sensemaking task: "At this point of your research you need to examine the concept of "Dynamic Queries"; you do not know where to start. A colleague of yours has given you a paper reference as a good starting point: a paper written by Shneiderman in 1996 and titled "Incremental ..." Your goal is to identify key researchers and publications that target this area and identify any commonalities or differences between these groups of people.." This version of the task description allowed participants to show how they thought about the literature domain, as well as how they used the tool to support their reasoning; these are the kinds of issue that matter for a CSII analysis. 
When instructing people on how to think aloud, it is important to emphasise that they are thinking aloud, not providing an explanation to the analyst. So people are not expected to articulate their thoughts in complete sentences, or to provide a running commentary on (for example) the design of the interface. It is generally helpful for participants to practice thinking aloud before starting the activity, if that is practical in the circumstances. Some participants will take to thinking aloud easily; others may need the occasional gentle reminder (e.g. "What are your thoughts now?").

Data from think-aloud can be analysed as described in section 3.3 to identify users' conceptual structures.

\subsection{Identifying system concepts}

Once a system design exists, it is possible to identify system concepts and compare them with user concepts. User concepts are identified as described above (through analysis of interview, Contextual Inquiry or Think Aloud data).

The main sources of system concepts are system descriptions and maybe a running system. Again, the data is analysed in whatever ways are possible (depending on what data sources are available) to identify core system concepts. In doing this analysis, one thing to avoid is extensive descriptions of interface widgets: rather, the analysis should focus on the underlying system representation. Interface widgets are a means to an end, not an end in themselves. For example, an analyst describing ALVi would focus on authors, publications, citations and the other concepts that users can access through the visualisations, not on the scroll-bars, tick boxes or search boxes.

\subsection{Identifying misfits}

Once suitable data has been gathered, misfits can be identified. The first step is simply to identify and compare system and user concepts; a second stage of analysis considers what actions are needed to change the system, and whether there are problems with actions. Misfits between user and system are the most important information-related misfits. These misfits fit into three classes, illustrated in Figure 11:

User concepts that are not represented within the system, and hence cannot be directly manipulated by the user ([a] in Figure 11). A very simple example related to ALVi is the concept of an idea. For example, when doing an evaluation study on ALVi, a participant gave this comment when asked whether publishing an influential article makes its author influential "...I think I would go for ideas...what it means actually it is not the paper but the ideas".

User concepts that are not represented in the system often force users to introduce workarounds, as users are unable to express exactly what they need to, and therefore use the system in a way it wasn't designed for. In these situations, users may adopt complementary tools, such as bits of paper or electronic notepads, to support their sensemaking. A well designed appropriation tool (such as those discussed above) can make such workarounds more seamless.

System concepts that the user has to know about but that are not naturally part of their initial understanding, and therefore need to be learned ([c] in Figure 11). An example 
involving information structures might be the ways that information is organised in a hierarchical classification system.

For users, these misfits may involve simply learning a new concept, or they may involve the users constantly tracking the state of something that has little significance to them.

An example of this was seen when we were evaluating ALVi. A few of the study participants conceptualised their literature domains differently from the majority (for whom the tool was tailored). One of these users was trying to adjust his personal sensemaking strategies to fit the tool's design. This user thought in terms of papers/titles and not in terms of authors; as a result, he kept getting confused as ALVi was designed with the authors at the centre of the sensemaking strategy. This user commented: "I am treating this as a visualization of papers rather than a visualization of authors".

User- and system concepts that are similar but non-identical, and which are often referred to by the same terms. This could be considered as an amalgamation of the two categories above (a user concept that the system doesn't represent and a system concept that the user has to know about), but has a particular set of implications, in terms of how the user has to mould their understanding to the system. For example, Blandford and Green [8] found that users of an electronic diary system had to adjust their concept of a "meeting" from being a relatively informal agreement between people to congregate in a particular place (which might involve a separate activity of booking a room) at a particular time to discuss agreed topics to being a more structured concept that typically did not include the purpose of the meeting, but did include an expectation of inviting rooms and other resources to participate in the meeting.

These misfits may cause difficulties because the user has to constantly map his / her natural understanding of the concept onto the one represented within the system, which may have a subtly different set of attributes that the user then has to work with.

As well as concepts being absent, some may be available but present some kind of difficulty. For users 'difficult' concepts are most commonly ones that are implicit - ideas they are aware of if asked but not ones they expect to work with. An example, for many people, is the end time of a meeting: in people's paper diaries, many engagements have start times (though these are often flagged as 'approximate' - e.g. '2ish') but few have end times, whereas electronic diaries (which are sold as diaries, but are better described as scheduling systems) force every event to have an end time (or a duration, depending on how you look at it). This forces users to make explicit information that they might not choose to. Of course, there are (typically busy) people for whom the "scheduling" nature of electronic diaries suits them better than the relatively imprecise structure of paper diaries [8], but these are a minority of users.

\subsection{Adding in information about actions}

The analyst can define how actions change the existence of entities or the values of attributes as a further step of analysis. The key question for sensemaking visualisation is whether or not the actions supported within the system are easily discovered and interpreted by users, whether they have unexpected side effects, and whether there are important action sequences that are longer or more tedious than necessary. 


\section{Discussion}

This chapter has focused on the design of sensemaking representations in terms of conceptual structures. Some sections (notably those on data gathering) address topics that are covered in more detail in other texts, and are included here for completeness. The heart of our argument is that sensemaking tools should where possible be transparent to their users, working as far as possible with people's existing conceptual representations, but that there is often additional benefit in introducing new, important and powerful concepts to people that allow them to think about the domain in a new way. Of course, to be included in a sensemaking tool, someone must have identified them as potentially valuable concepts to include. The sections on requirements and evaluation have focused particularly on conceptual fit between a typical user and a system.

Recognising that every user has individual interests and ways of thinking about a domain, we have also argued that appropriation tools such as features for annotating and marking representations can help individual users to make sense of a domain on their own terms, and to track their own developing understanding.

In the design section, we have proposed a set of representation types that might support users' sensemaking in different domains. We do not claim that this set is comprehensive, and we look forward to discovering (or generating) novel representation types for new sensemaking problems. This set has been proposed based on a review of the interactive visualisation literature and our own experience. A particular sensemaking tool may bring together multiple representations.

Having an appropriate conceptual structure is not the only success criterion for an interactive visualisation. Many other usability and usefulness criteria will also come into play. Conceptual structure is, nevertheless, an important contributor to usability and utility that has, historically, received insufficient attention in design and evaluation of interactive visualisations for sensemaking.

\section{References}

1. Ahlberg, C., \& Shneiderman, B. (1994). Visual Information Seeking Using the FilmFinder. CHI'94.

2. Attfield S. \& Blandford A. (2010) Discovery-led Refinement in e-Discovery Investigations: Sensemaking, Cognitive Ergonomics and System Design. Artificial Intelligence and Law - Special Issue on e-Discovery, 18.4, 387-412.

3. Attfield, S. \& Blandford, A. (2011) Making sense of digital footprints in team-based legal investigations: The acquisition of focus. Human-Computer Interaction Journal. $26.1 \& 2,38-71$

4. Barone, R., \& Cheng, P. C. H. (2004). Representations for Problem Solving: On the Benefits of Integrated Structure. In Proc. IV04. IEEE Computer Society (pp. 575-580)

5. Bertin, J. (1983). Semiology of Graphics: Diagrams, Networks, Maps. University of Wisconsin Press

6. Beyer, H., Holtzblatt, K. (1998) Contextual Design. San Francisco : Morgan Kaufmann

7. Blandford, A. \& Attfield, S. (2010) Interacting with information. Morgan \& Claypool. http://www.morganclaypool.com/doi/abs/10.2200/S00227ED1V01Y200911HCI006

8. Blandford, A. E. \& Green, T. R. G. (2001) Group and individual time management tools: what you get is not what you need. Personal and Ubiquitous Computing. 5.4, 213 230. 
9. Blandford, A., Green, T. R. G., Furniss, D. \& Makri, S. (2008) Evaluating system utility and conceptual fit using CASSM. International Journal of Human-Computer Studies. 66. 393-409.

10.Carroll, J., Howard, S., Vetere, F., Peck, J., \& Murphy, J. (2002). Just what do the youth of today want? Technology appropriation by young people. In Proc. 35th Annual Hawaii International Conference, 1777-1785.

11.Carroll, J. M. \& Rosson, M. B. (1992) Getting around the task-artifact cycle: how to make claims and design by scenario. ACM Transactions on Information Systems, 10.2, 181-21.

12.Craft, B., \& Cairns, P. (2008). Directions for Methodological Research in Information Visualisation. In Information Visualisation, 2008. 44-50.

13.Dix, A. (2007). Designing for Appropriation. In Proc. 21st British HCI Group Conference (Vol. 2, pp. 28-30). Lancaster, UK

14.Ericsson, K.A. \& Simon, H.A. (1984) Protocol analysis: Verbal reports as data. Cambridge, MA: MIT Press.

15.Faisal, S., Attfield, S. \& Blandford, A. (2009) A Classification of Sensemaking Representations. CHI 2009 Workshop on Sensemaking.

16.Faisal, S., Cairns, P. \& Blandford, A. (2006) Developing User Requirements for Visualizations of Literature Knowledge Domains. In Proc. IV06.

17.Faisal, S., Cairns, P. \& Blandford, A. (2007) Building for Users not for Experts: Designing a Visualization of the Literature Domain. In Proc. IV07.

18.Faisal, S., Cairns, P. \& Blandford, A. (submitted) Designing for seamless interaction with visual analytic tools. Submitted for journal publication.

19.Fekete, J., Grinstein, G., \& Plaisant, C. (2004). IEEE InfoVis 2004 Contest, the history of InfoVis. . Retrieved from www.cs.umd.edu/hcil/iv04contest (2004).

20.Hackos, J. \& Redish, J. (1998) User and Task Analysis for Interface Design. John Wiley \& Sons

21.Heer, J., \& Boyd, D. (2005). Vizster: Visualizing Online Social Networks. InfoVis'05.IEEE

22.Johnson, J. \& Henderson, A. (2012) Conceptual Models: Core to Good Design. Synthesis Lectures on Human-Centered Informatics. Morgan \& Claypool Publishers. doi: 10.2200/S00391ED1V01Y201111HCI012

23.Kang, Y., Görg, C. \& Stasko, J. (2011) How Can Visual Analytics Assist Investigative Analysis? Design Implications from an Evaluation, IEEE Transactions on Visualization and Computer Graphics, 17.5, 570-583.

24.Klein, G., Phillips, J. K., Rall, E. L., \& Peluso, D. A. (2007). A Data-Frame Theory of Sensemaking. In R. Hoffman (Ed.), Expertise Out of Context: Proc. Int. Conf. on Naturalistic Decision Making. Lawrence Erlbaum Assoc. Inc, US, 113-155.

25.Kuniavsky, M. (2003). Observing the user experience: a practitioner's guide to user research, Morgan Kaufmann, San Francisco.

26.Moran, T.P. (1983) Getting Into a System: External-internal Task Mapping Analysis, in A.Janda (ed.), Human Factors in Computing Systems, pp.45-49. ACM SIGCHI and Human Factors Society conference proceedings, Boston, December 1983. New York: ACM Press.

27.Norman, D.A. (1986) Cognitive Engineering. In D.A. Norman and S.W. Draper, Eds. User Centered System Design, Hillsdale NJ: Lawrence Erlbaum, 31-62

28.Payne, S.J., Squibb, H.R. \& Howes, A. (1990) The nature of device models: The yoked state space hypothesis, and some experiments with text editors. Human-Computer Interaction, 5, 415-444.

29.Pirolli P. and Card S., (2005) The Sensemaking Process and Leverage Points for Analyst Technology as Identified Through Cognitive Task Analysis. In: Proc. International Conference on Intelligence Analysis (McLean, VA, May 2-6, 2005). 
30.Russell, D. M., Stefik, M., Pirolli, P., \& Card, S. (1993). The Cost Structure of Sensemaking. In Proc. INTERACT '93 and CHI '93 (pp. 269-276). Amsterdam, The Netherlands: ACM

31.Shneiderman, B. (1996). The Eyes Have It: A Task by Data Type Taxonomy for Information Visualisations. In Proceedings of the IEEE Symposium on Visual Languages (p. 336-343). IEEE Computer Society

32.Shrinivasan, Y. B., \& Wijk, J. J. V. (2008). Supporting the analytical reasoning process in information visualisation. In Proc. CHI (pp. 1237-1246). ACM

33.Spence, R. (2007). Information Visualisation: Design for Interaction (2nd ed.). ACM Press Books

34.Stasko, J., Görg, C. \& Liu, Z. (2008) Jigsaw: Supporting Investigative Analysis through Interactive Visualization, Information Visualization, 7.2, 118-132.

35.Stasko, J., Gorg, C., Liu, Z., \& Singhal, K. (2008). Jigsaw: supporting investigative analysis through interactive visualisation. Information Visualisation, IEEE , 7(2), 118

36. Toulmin, S. (1958). The Uses of Argument, Cambridge, England: Cambridge University Press.

37.Tufte, E. R. (1986). The Visual Display of Quantitative Information. Graphics Press Cheshire, CT, USA

38.Uren, V., Buckingham Shum, S., Bachler, M., \& Li, G. (2006). Sensemaking tools for understanding research literatures: Design, implementation and user evaluation. IJHCS, 64(5), 420-445.

39.Ware, C. (2004). Information Visualisation: Perception for Design. Morgan Kaufmann 40.Wigmore, J.H. (1931). The Principles of Judicial Proof. Little, Brown \& Co.

41.Williamson, C. \& Shneiderman, B. (1992) The Dynamic HomeFinder: Evaluating dynamic queries in a real-estate information exploration system, Proc. ACM SIGIR'92. 338-346. 